\title{
Theoretical prediction of Reynolds stresses and velocity profiles for barotropic turbulent jets
}

\author{
Eric Woillez ${ }^{*}$ and Freddy Bouchet ${ }^{\dagger}$ \\ Univ Lyon, Ens de Lyon, Univ Claude Bernard, \\ CNRS, Laboratoire de Physique, F-69342 Lyon, France
}

(Dated: November 12, 2018)

\begin{abstract}
It is extremely uncommon to be able to predict the velocity profile of a turbulent flow. In twodimensional flows, atmosphere dynamics, and plasma physics, large scale coherent jets are created through inverse energy transfers from small scales to the largest scales of the flow. We prove that in the limits of vanishing energy injection, vanishing friction, and small scale forcing, the velocity profile of a jet obeys an equation independent of the details of the forcing. We find another general relation for the maximal curvature of a jet and we give strong arguments to support the existence of an hydrodynamic instability at the point with minimal jet velocity. Those results are the first computations of Reynolds stresses and self consistent velocity profiles from the turbulent dynamics, and the first consistent analytic theory of zonal jets in barotropic turbulence.
\end{abstract}

Theoretical prediction of velocity profiles of inhomogeneous turbulent flows is a long standing challenge, since the nineteenth century. It involves closing hierarchy for the velocity moments, and for instance obtaining a relation between the Reynolds stress and the velocity profile. Since Boussinesq in the nineteenth century, most of the approaches so far have been either empirical or phenomenological. Even for the simple case of a three dimensional turbulent boundary layer, plausible but so far unjustified similarity arguments may be used to derive von Kármán logarithmic law for the turbulent boundary layer (see for instance [1]), but the related von Kármán constant 2] has never been computed theoretically. Still this problem is a crucial one and has some implications in most of scientific fields, in physics, astrophysics, climate dynamics, and engineering. Equations (677), (9), and (11) are probably the first prediction of the velocity profile for turbulent flows, and relevant for barotropic flows.

In this paper we find a way to close the hierarchy of the velocity moments, for the equation of barotropic flows with or without effect of the Coriolis force. This two dimensional model is relevant for laboratory experiments of fluid turbulence [3, liquid metals 4], plasma [5], and is a key toy model for understanding planetary jet formation [6] and basics aspects of plasma dynamics on Tokamaks in relation with drift waves and zonal flow formation [7. It is also a relevant model for Jupiter troposphere organization [8. Moreover, our approach should have future implications for more complex turbulent boundary layers, which are crucial in climate dynamics in order to quantify momentum and energy transfers between the atmosphere and the ocean.

It has been realized since the sixties and seventies in the atmosphere dynamics and plasma communities that in some regimes two dimensional turbulent flows are

\footnotetext{
* Eric.Woillez@ens-lyon.fr

$\dagger$ Freddy.Bouchet@ens-lyon.fr
}

strongly dominated by large scale coherent structures. Jets and large vortices are often observed in numerical simulations or in experiments, but the general mechanism leading to such an organization of the flow at large scales is subtle and far from being understood. For simplicity, we consider in this paper the case of parallel jets favored by the $\beta$ effect, however without $\beta$ effect both jets and vortices can be observed [4, 9, 10]. When a large scale structure is created by the flow, a quasilinear approach may be relevant. Such a quasilinear approach requires solving a coupled equation for the mean flow and the Lyapunov equation that describes the fluctuations with a Gaussian approximation, just like the Lenard-Balescu equation in plasma kinetic theory. Numerical approaches and theoretical analysis have been systematically developed for fifteen years in order to solve and study such quasilinear or related approximations [6, 11. In a recent theoretical paper, the range of validity of such an approach has been established by proving the self consistency of the approximations of weak forcing and dissipation limit [12]. While this work gave theoretical ground to the approach, explicit formula for the Reynolds stress cannot be expected in general. However, in a recent work [13, an expression for the Reynolds stress has been derived from the momentum and energy balance equations by neglecting the perturbation cubic terms in the energy balance (this follows from the quasilinear approach justification 12), but also neglecting pressure terms (not justified so far, see [14]). This approach surprisingly predicts a constant velocity profile for the outer region of a large scale vortex in two dimensions that does not depend on the detailed characteristics of the stochastic forcing but only on the total energy injection rate $\epsilon$ expressed in $\mathrm{m}^{2} \mathrm{~s}^{-3}$. Another analytic expression for the Reynolds stress has also been derived in the particular case of a linear velocity profile $U$ in [15. For the case of dipoles for the 2D Navier-Stokes equations, the papers [16, 17] following a computation analogous to the one given in [15], shows that if the vorticity is passively advected (the third term in equation (5) below is neglected), then the expression for the Reynolds stress discussed in 
[10] is recovered ( [17] also discusses other interesting aspects related to parts of the flow for which this relation is not correct). What are the criteria for the validity of these results? Can we reconcile the different results giving a full theoretical justification and extend these for more general cases?

We start from the equations for a barotropic flow on a periodic beta plane with stochastic forcing

$$
\partial_{t} \mathbf{V}+\mathbf{V} \cdot \nabla \mathbf{V}=-r \mathbf{V}-\frac{1}{\rho} \nabla P+\beta_{d} y\left(\begin{array}{c}
V_{y} \\
-V_{x}
\end{array}\right)+\sqrt{2 \epsilon} \mathbf{f}
$$

where $\mathbf{V}:=\left(\begin{array}{c}V_{x} \\ V_{y}\end{array}\right)$ is the two dimensional velocity field with $\nabla \mathbf{V}=0$. $\quad r$ models a linear friction, and $\mathrm{f}$ is a stochastic force white in time, with energy injection rate $\epsilon, \beta_{d}$ is the Coriolis parameter, $y$ the north-south coordinate. Following [12] we choose time and space units such that the mean kinetic energy is 1 , and $L_{x}=1$. The non dimensional equations for the vorticity $\Omega=\nabla \wedge \mathbf{V}$ are

$$
\partial_{t} \Omega+\mathbf{V} . \nabla \Omega=-\alpha \Omega-\beta V_{y}+\sqrt{2 \alpha} \eta
$$

where $\eta=\nabla \wedge f$, and $\mathbf{V}$ denotes from now on the nondimensional velocity. Now $\alpha=L \sqrt{\frac{r^{3}}{\epsilon}}$ is a nondimensional parameter although we will often refer to it as the "friction". $\beta=\sqrt{\frac{r}{c}} L^{2} \beta_{d}$ is the nondimensional Coriolis parameter. Eq. (2) still has three nondimensional parameters, $\alpha, \beta$ and $\vec{K}$, the typical Fourier wavenumber where energy is injected.

Neglecting the pressure and cubic terms in the energy balance and enstrophy balance, it is straightforward to obtain the Reynolds stress expression

$$
\langle u v\rangle=\frac{\epsilon}{U^{\prime}}
$$

where $U^{\prime}=\mathrm{dU} / \mathrm{dy}$. This generalizes the result obtained for a vortex [13] to the case of a jet with mean velocity $U$. Is it possible to justify those hypothesis on theoretical ground, uncover the validity range of (3), and to generalize it? We note that detailed numerical studies of the energy balanced has been discussed in several papers [10, 18, 19.

In order to derive eq. (3), the key idea is to use the already justified [12] quasilinear approximation in the limit of small forces and friction (inertial regime, $\alpha \ll 1$ ), and to further consider the limit of small scale forcing $(K \gg 1)$, with fixed $\beta$. In these limits, energy is injected at small scale and is dissipated at the largest scale of the flow. $\alpha \ll 1$ is the proper regime for most geophysical turbulent flows, for instance for giant gaseous planets like Jupiter [20, 21], and many two dimensional or rotating turbulence experiments. The small scale forcing limit $K \gg 1$ is the most common framework for turbulence studies (see for ex. 14]) and relevant for Jupiter troposphere. Also, computing the pressure from the NavierStokes equations involves inverting a Laplacian. It is thus natural to expect the pressure term to have a power expansion in the parameter $\frac{1}{K}$, and thus vanish in the limit of large $K$. The main idea is then to separate the flow $\mathbf{V}$ in two parts, $\mathbf{V}(\mathbf{r}, t)=U(y, t) \mathbf{e}_{x}+\left(\begin{array}{c}u(\mathbf{r}, t) \\ v(\mathbf{r}, t)\end{array}\right)$. The mean velocity $U(y) \mathbf{e}_{x}=\frac{1}{L_{x}} \int \mathrm{d} x \mathbb{E}[\mathbf{V}(x, y)]$ called the mean flow or zonal flow, is defined as both the zonal and stochastic average of the velocity field. In the following, the bracket \langle\rangle will be used for this zonal and stochastic average. We are left with two coupled equations, one governing the dynamics of the mean flow, the other one describing the evolution of eddies. In the limit where $\alpha$ is small, it has been proven that fluctuations are of order $\sqrt{\alpha}$ and thus it is self-consistent to neglect nonlinear terms in the equation for fluctuations [12. Then one can justify [12] that, at leading order in $\alpha$, the full velocity field statistics are described by a quasi-Gaussian field (the velocity field is not Gaussian, but the marginals when the zonal flow is fixed are Gaussian, justifying a posteriori a second order closure corresponding to the quasilinear approximation). Using also the incompressibility condition, we obtain the quasilinear model

$$
\begin{gathered}
\partial_{t} U=-\alpha\left[\partial_{y}\langle u v\rangle+U\right] \\
\partial_{t} \omega+U \partial_{x} \omega+\left(\beta-U^{\prime \prime}\right) v=-\alpha \omega+\eta
\end{gathered}
$$

where we have introduced $\omega=\partial_{x} v-\partial_{y} u=\Delta \psi$, the vorticity of the fluctuations. Eq. (4) shows that the typical time scale for the evolution of the mean flow $U$ is $\frac{1}{\alpha}$ which is, following our assumption $\alpha \ll 1$, much larger than the time scale for the evolution of eddies. Using this time scale separation, we will consider that $U$ is a constant field in the second Eq. (5), and we will always solve $\omega(t)$ for a given $U$. We follow the strategy:

- First we solve the linear Eq. (5) and compute the stationary distribution $\left\langle\omega^{2}\right\rangle$ as a functional of $U$.

- The enstrophy balance for the fluctuations allows us to relate $\left\langle\omega^{2}\right\rangle$ to the divergence of the Reynolds stress tensor (see the Supplementary Material in appendix).

- Last we can use this expression to close the first Eq. (4), and discuss possible stationary profiles $U$.

To reach the first objective, we take advantage of the asymptotic regimes $\alpha \rightarrow 0$ and $K \rightarrow \infty$. When we take those two limits, it is natural to ask whether they commute or not, and which nondimensional parameter will govern the difference between $\alpha \rightarrow 0$ first or $K \rightarrow \infty$ first. Our asymptotic calculations show that the key parameter is the ratio between $\frac{U^{\prime \prime}}{K}$ and $\alpha$. Taking the limit $\alpha \rightarrow 0$ first amounts to saying that $\frac{U^{\prime \prime}}{\alpha K}$ is very large.

We first take the limit $K \rightarrow \infty$ while keeping $\alpha$ small but finite. The idea is to write Eq. (5) in an integral form using the Green function of the Laplacian, and use the fact that the Green function decreases very fast when $K$ is large, which implies that the evolution of the flow is local in space. At this stage of the calculation, $\alpha$ is small but finite, and the expression for the Reynolds stress depends both on $\alpha$ and on the properties of the stochastic 
forcing. The complete calculation is reported in the Supplementary Material [? ]. We emphasize that as long as $\alpha$ is kept finite, the Reynolds stress depends on the Fourier spectrum of the stochastic forcing $\eta$. The result shows that the Reynolds stress can be expressed analytically as

$$
\langle u v\rangle=\frac{1}{2 \alpha} \chi\left(\frac{U^{\prime}}{2 \alpha}\right),
$$

where the explicit expression of $\chi$ is a parametric integral, see [? ]. The stationary profile $U$ thus verifies $\frac{U^{\prime \prime}}{2 \alpha} \frac{1}{2 \alpha} \chi^{\prime}\left(\frac{U^{\prime}}{2 \alpha}\right)=-U$, which can be integrated using a primitive $X$ of the function $x \rightarrow x \chi^{\prime}(x)$ in

$$
X\left(\frac{U^{\prime}}{2 \alpha}\right)+\frac{1}{2} U^{2}=C,
$$

where $C$ is the integration constant. It is in itself remarkable that for some range of the parameters, the flow of the barotropic quasilinear model can be computed from a Newtonian equation like (7).

In Eq. (7), $X$ plays the role of a potential as if the equation would describe a particle moving in a onedimensional potential. The constant in the right-hand side is set by $U^{\prime}(0)$ and depending on the value of this constant, there can be one, two or three solutions as shown in Fig. (1). If $C>X_{\max }$, there is one solution for which $U$ never vanishes. As the total flow momentum is zero, such solutions with either $U>0$ or $U<0$ are not physical. If $X_{0}<C<X_{\max }$, there are three possible solutions, one is periodic, the other two diverge. The periodic solution corresponds to $\frac{U^{\prime}}{2 \alpha}$ confined in the well of $X$. In that case the flow is periodic and the solution exchanges kinetic energy in the term $\frac{1}{2} U^{2}$ with potential energy $X\left(\frac{U^{\prime}}{2 \alpha}\right)$. Outside the well, the solutions are diverging, one corresponds to an increasing $U$ and the other to a decreasing $U$. A linear stability analysis of the periodic solution of (7) shows that this solution is unstable whereas the diverging solution is stable. Thus, the periodic regular solution is not a suitable candidate for the stationary mean velocity profile $U(y)$. If we now take the limit of vanishing $\alpha$ in expression (6), a straightforward calculation using the explicit expression of $\chi$ (given in [? ]) allows us to recover expression (3) for the Reynolds stress because $U^{\prime}\langle u v\rangle \underset{\alpha \rightarrow 0}{\longrightarrow} 1$ which is Eq. 3 with dimensional units. The physical interpretation of the limiting case (3) is very enlightening. The term $U^{\prime}\langle u v\rangle$ can be interpreted as the rate of energy transferred from small scale to large scale, therefore expression (3) is consistent: with the limit of large $K$, the evolution of eddies becomes local as if the perturbation only sees a region of width $\frac{1}{K}$ around itself, and thus the different parts of the flow are decoupled. The other limit of small $\alpha$ forces the energy to go to the largest scale to be dissipated because the dissipation at small scales becomes negligible.

Let us study the other limit where $\alpha$ goes to zero first. The techniques used in this second case are very different than the previous one. We assume in this section that the linearized dynamics has no unstable modes. The calculation involves Laplace transform tools that were used

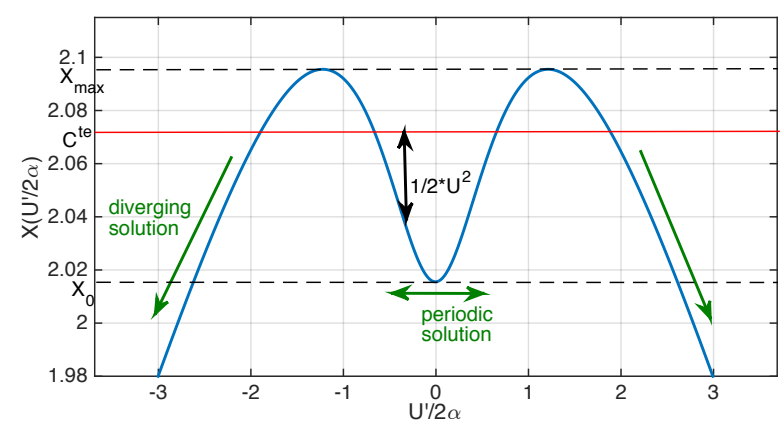

FIG. 1: In the limit of small scale forcing, the mean flow can be computed analytically from the Newtonian Eq. (7). We show here that the situation is analogous to a particle moving in a one-dimensional potential. The blue curve displays the potential $X$ appearing in Eq. (7). If the constant of motion is less than $X_{\max }$, there are two classes of solutions: the solution can be confined in the central well and is thus regular and periodic in space, or it is outside the well, and in this latter case it diverges.

in 22 to study the asymptotic stability of the linearized Euler equations. In the limit $\alpha \ll 1$, using [12, we derive in the Supplementary Material [? ] the relation between the Reynolds stress divergence and the long time behavior of a disturbance $\omega(y, 0)$ carried by a mean flow $U(y)$. This is an old problem in hydrodynamics, one has to solve the celebrated Rayleigh equation

$$
\left(\frac{d^{2}}{d y^{2}}-k^{2}\right) \varphi_{\delta}(y, c)+\frac{\beta-U^{\prime \prime}(y)}{U(y)-c-i \delta} \varphi_{\delta}(y, c)=\frac{\omega(y, 0)}{i k(U(y)-c-i \delta)},
$$

where $\varphi_{\delta}(y, c):=\int_{0}^{\infty} \mathrm{d} t \psi(y, t) e^{-i k(c+i \delta) t}$ is the Laplace transform of the stream function, and $k$ is the $x$ component of the wavevector. The Laplace transform $\varphi_{\delta}$ is well defined for any non zero value of the real variable $\delta$ with a strictly negative product $k \delta$. $c$ has to be understood as the phase speed of the wave, and $k \delta$ is the (negative) exponential growth rate of the wave. Involved computations are then required to give the explicit expression of the Reynolds stress. Let us just mention that the difficulty comes from the fact that we have to take the limit $\delta \rightarrow 0$ first in Eq. (8) before $K \rightarrow \infty$. Then we can relate $\omega(y, \infty)$ to the Laplace transform $\varphi_{\delta}$ taken at $\delta=0$. The expression for the Reynolds stress in the inertial limit involves an integral with the profile $U$ in the denominator of the integrand. The integral is defined only in regions of the flow where $U^{\prime}$ does not vanish, or to state it more precisely, where the parameter $\frac{K U^{\prime}}{U^{\prime \prime}}$ is large. Therefore, there exists a small region of size $\frac{1}{K}$ around the maximum where the asymptotic expansion breaks down. In the outer region away from the extrema, we recover the expression (3) in the inertial limit $\alpha \rightarrow 0$ first. For strictly monotonic velocity profiles $U$, we conclude that the inertial limit and the small scale forcing limit commute and that expression (3) is expected to be valid. 


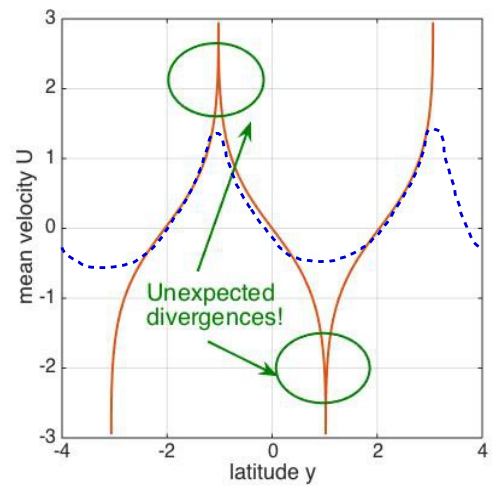

(a)
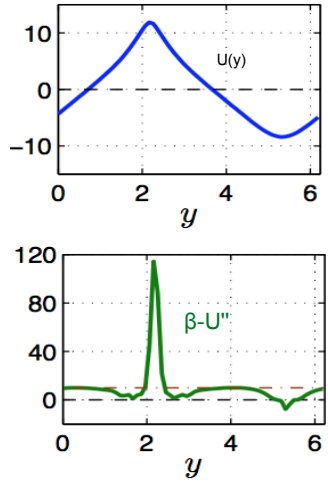

(b)
FIG. 2: (a) Jet profiles obtained as an analytic solution of (9) (red curve) together with a qualitative real velocity profile (blue curve). (b) Real velocity profile obtained in numerical simulations 23 . The analytic solution for $U$ has divergences at the extrema that are regularized by a cusp at the eastward jet and a parabola at the westward jet.

Using expression (3) for the Reynolds stress, we can solve Eq. (4) for the stationary profile. It writes

$$
\frac{\mathrm{d}}{\mathrm{d} y}\left(\frac{\epsilon}{U^{\prime}}\right)+U=0
$$

Whatever the value of the free parameter $U^{\prime}(0)$, all profiles $U$ are diverging in finite length. An example of such a profile $U$ is given in Fig. (2a). In red, we have plotted different solutions of Eq. (9) together such that the mean velocity profile $U$ is composed of many diverging jets. In blue, we have drawn at hand what we expect qualitatively from a real velocity profile. An example of a real profile obtained by numerical simulations in 23 is displayed in Fig. (2b). The fact that Eq. (9) predicts diverging profiles $U$ shows that the expression for the Reynolds stress (3) is not valid everywhere in the flow, but it holds only in the spatial subdomains where the flow is monotonic, not at the extrema. We observe that both divergences are regularized, by a cusp at the eastward jet maximum, and by a parabolic profile at the westward jet minimum. The second aim of this paper is to explain the asymmetric regularization of the eastward and westward jets.

Numerical simulations like the one performed in Fig. (2b) show that the mean velocity profile is regularized at a very small scale at its maximum. As we explained previously, there exists a region of typical size $\frac{1}{K}$ around the maximum where the asymptotic expansion for the Reynolds stress breaks down. It is thus natural to choose the ansatz $\widetilde{U}(y):=U\left(\frac{y}{K}\right)$. The scaling in $\frac{1}{K}$ implies that the ratio $\frac{U^{\prime \prime}}{K \alpha}$ is very large at the cusp because $U^{\prime \prime} \propto$ $K^{2}$. The cusp is then described by the inhomogeneous Rayleigh Eq. (8). If we put the ansatz $\widetilde{U}$ in $(8)$ and

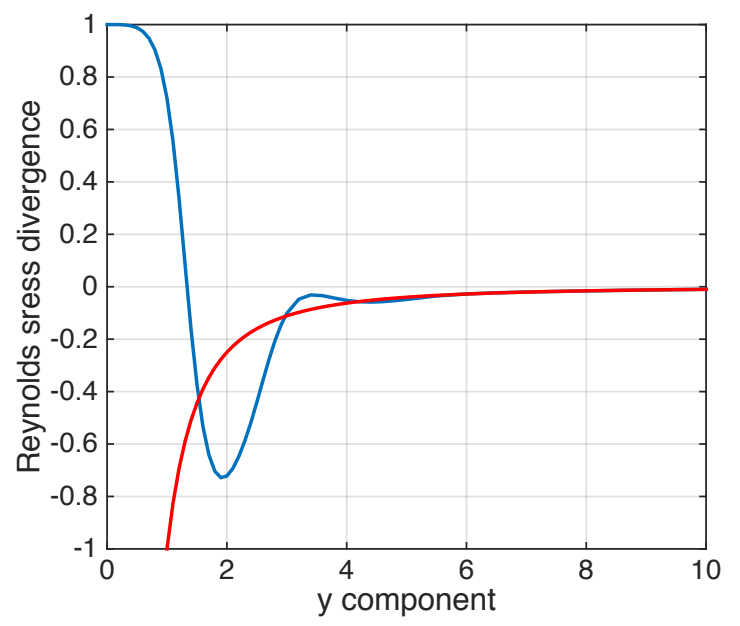

FIG. 3: The Reynolds stress divergence $-\partial_{y}\langle u v\rangle$ (blue curve) computed for a parabolic profile $\widetilde{U}(y)=-\frac{y^{2}}{2}$ around its maximum $y=0$. It is satisfying to observe that the red curve $\frac{\widetilde{U}^{\prime \prime}}{\widetilde{U}^{\prime 2}}$ matches the blue curve for large $y$ according to expression 3 .

consider the limit of large $K$, we get

$$
\left(\frac{d^{2}}{d y^{2}}-\tan ^{2} \theta\right) \varphi_{\delta}(y, c)-\frac{\tilde{U} "(y)}{\tilde{U}(y)-c-i \delta} \varphi_{\delta}(y, c)=\frac{e^{i \sin \theta y}}{\tilde{U}(y)-c-i \delta},
$$

where $\cos \theta=\frac{k}{K}$. The solution of this equation with $\delta \rightarrow 0$ gives us the Laplace transform of the stream function with which we can express the Reynolds stress. The $\beta$-effect disappears completely from the equation of the cusp because in the region of the cusp, the curvature is so large that it overcomes completely the $\beta$-effect, as can be seen on the green curve of Fig. 2b). Eq. 10p together with the equations linking the Reynolds stress with $\varphi$ have a numerical solution, which proves that our scaling $\widetilde{U}(y):=U\left(\frac{y}{K}\right)$ is self-consistent. This solution can not be expressed analytically and depends on the Fourier spectrum of the stochastic forcing. An example of a numerical integration of the Reynolds stress divergence $-\partial_{y}\langle u v\rangle$ for the cusp is displayed in Fig. (3) for $\widetilde{U}(y)=-\frac{y^{2}}{2}$ and a stochastic forcing with a semi annular Fourier spectrum where $\theta$ ranges between $-\frac{\pi}{3}$ and $\frac{\pi}{3}$. In Fig. 3 , the red curve is the asymptote $\frac{\widetilde{U}^{\prime \prime}}{\widetilde{U}^{\prime 2}}$ obtained from formula (3) with $\epsilon=1$. When a jet is in a stationary state, the cusp profile joins smoothly the outer region of the jet where the result (3) is valid.

Another physical phenomenon at the maximum of the jet is called "depletion at the stationary streamlines" and has first been observed in 22. It means that at critical latitudes where $U^{\prime}=0$, any vorticity perturbation of the flow $\omega_{0}$ has to asymptotically vanish with time. One main consequence of this phenomenon is the relation

$$
U\left(y_{c r}\right)=-\frac{\epsilon K^{2}}{r U^{\prime \prime}\left(y_{c r}\right)},
$$


where $y_{c r}$ is the latitude of the extremum. From (11) we learn that, even if the velocity profile of the cusp depends on the details of the forcing, the maximal curvature of the profile satisfies a more general relation, and it would therefore be very interesting to check it in full numerical simulations with different types of forcing spectra.

The previous discussion successfully explained the jet regularization of the eastward jet cusp. As clearly observed in Fig. (2b), and from Jupiter data, westward jets do not produce cusps. At first sight, it may seem that all the theoretical arguments used so far, the energy balance, the asymptotic expansions, and the results (3) and (11), do not break the symmetry between eastward and westward jets, as $\beta$ disappears from all these computations. However, as clearly stressed in [12, the whole theoretical approach relies on an assumption of hydrodynamic stability for $U$. The asymmetry is clearly visible in the Rayleigh-Kuo criterion, that states that when $\beta-U^{\prime \prime}$ change sign, an instability may develop. We will now argue that the turbulent flow is constantly oscillating between a stable and unstable solution, in order to control the westward jet behavior. This means that the flow is not linearly stable, but only marginally stable. As shown in the following, the instability is localized at the extremum of the westward jet, and the unstable mode has a very small spatial extension. That's why the flow can be considered as stable away from the westward extremum of the jet and the assumptions of expression (3) are satisfied.

To check this marginal stability hypothesis, we solved numerically the homogeneous PDE for a perturbation carried by a mean flow

$$
\partial_{t} \omega+i k U \omega+i k\left(\beta-U^{\prime \prime}\right) \psi=0 .
$$

We chose a parabolic mean velocity with a small perturbation at its minimum $U(y)=\gamma \frac{y^{2}}{2}-\eta e^{-\frac{y^{2}}{\sigma^{2}}}$. The main curvature $\gamma$ is chosen to be slightly smaller than $\beta$, and we performed simulations using different values of $\eta$ and $\sigma$. The result of one of those simulations is displayed in figure (4). The red curve shows the Rayleigh-Kuo criterion $\beta-U^{\prime \prime}$ which is locally violated around $y=0$. The blue curve shows the amplitude of the perturbation $\left|\omega^{2}(y, t)\right|-1$ from which we can then express the Reynolds stress divergence. We check that the growth rate of the perturbation is indeed exponential with time with a complex rate $c$.

In this paper we gave a global consistent picture of how a zonal jet is sustained in a steady state through continuous energy transfer from small scale to large scale. Eq. (7) and (9) are probably the most striking results of this work, showing for the first time that it is possible to find closed equations for the velocity profile of turbulent flows. It illustrates that, although far from equilibrium, turbulent flow velocity profiles may be described by self consistent equations, as density of other macroscopic profiles can be described in condensed matter physics. This is a fundamental property which existence is far from

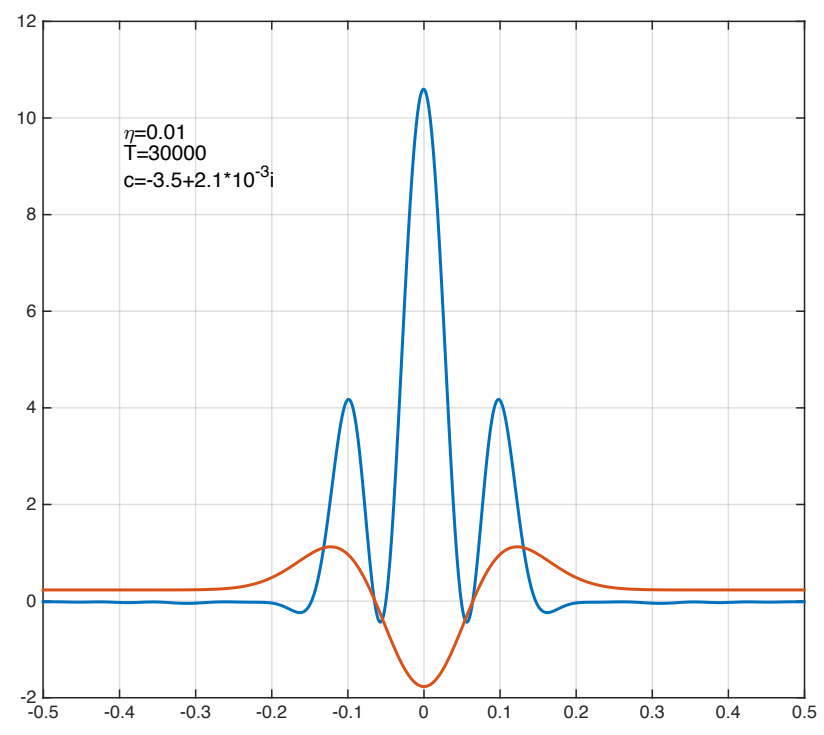

FIG. 4: Amplitude of the perturbation $\left|\omega^{2}(y, t)\right|-1$ (blue curve) carried by a mean flow $U$. The Rayleigh-Kuo criterion $\beta-U^{\prime \prime}$ is displayed in red. The simulation shows that the perturbation grows exponentially with the complex rate $c$.

obvious, and that no other approach was able to establish so far. In this paper, we have considered the case of a Rayleigh friction as the mechanism for removing energy that is transferred to the largest scale. Rayleigh friction is a rather ad-hoc type of damping (although it can be justified in certain cases involving, e.g., Ekman pumping). If one would consider other kinds of friction, for instance scale-selective damping, provided that this damping actually acts on the largest scales of the flow, in a corresponding inertial limit we expect most of our results to easily generalize (the proper dissipation operator should then replace Rayleigh friction in equation (4)). Indeed the processes that explain the computation of the Reynolds stress are inertial in nature and independent from the dissipation mechanisms. As an example, the case of viscous dissipation has been discussed in section 5.1.2 of [12], showing that while the regularization by viscosity is very different from the regularization by linear friction, the inertial results for the Reynolds stresses do coincide. This is true as far as the shear is non zero and equation (3) is concerned; by contrast the regularization of the cusp is dissipation dependent. In some specific cases, changing the dissipation mechanism may induce specific instability modes, like boundary layer modes due to viscous dissipation, however such effects are expected to be non generic. Extension and generalizations of our approach can be foreseen for other geometries (on the sphere), for more comprehensive quasi-geostrophic models of atmosphere jets, and for classes of flows dominated by a strong mean jet, for instance in some instances of boundary layer theory. 


\section{ACKNOWLEDGMENTS}

We thank P. Ioannou for interesting discussion during the preliminary stage of this work. The research leading to these results has received funding from the European Research Council under the European Union's seventh Framework Program (FP7/2007-2013 Grant Agreement No. 616811).

\section{Appendix A: Computation of the Reynolds stress in the small scale forcing limit}

This section is devoted to the computation of the Reynold's stress $\langle u v\rangle$ using the stochastic equation for the fluctuations

$$
\partial_{t} \omega+U \partial_{x} \omega+\left(\beta-U^{\prime \prime}\right) v=-\alpha \omega+\eta,
$$

which is equation (5) in the article. The noise $\eta$ is white in time and has spatial correlation function $C$. Using the Ito convention for stochastic calculus, it is straightforward to obtain the enstrophy balance for the fluctuations

$$
\frac{1}{2} \partial_{t}\left\langle\omega^{2}\right\rangle+\left(\beta-U^{\prime \prime}\right)\langle v \omega\rangle=-\alpha\left\langle\omega^{2}\right\rangle+\frac{1}{2} C(0),
$$

and thus in statistical stationary state we get the very useful relation

$$
\langle v \omega\rangle=\frac{1}{U^{\prime \prime}-\beta}\left[\alpha\left\langle\omega^{2}\right\rangle-\frac{1}{2} C(0)\right] .
$$

Thanks to the incompressibility condition, $\langle v \omega\rangle$ is related to the Reynolds stress through $\langle v \omega\rangle=-\partial_{y}\langle u v\rangle$.

First we can take advantage of the invariance along the $x$ direction by taking the Fourier transform of (A1) in $x$. The Fourier transform in $y$ does not provide an obvious simplification as the profile $U$ depends on $y$. However, we can use the linearity to express the solution as the sum of particular solutions for independent stochastic forcings $\eta_{l}(y, t)$. Each of these forcings has a correlation function $c_{l}(y)=e^{i l y}$, this means that we have the relation $\mathbb{E}\left[\eta_{l}(y, t) \eta_{l}\left(y^{\prime}, t\right)\right]=e^{i l\left(y-y^{\prime}\right)} \delta\left(t-t^{\prime}\right)$. We take the Fourier transform in $x$ defined by $\omega_{k}(y):=\frac{1}{L_{x}} \int \mathrm{d} x \omega(x, y) e^{-i k x}$ with $k$ taking the values $\frac{2 \pi}{L_{x}} n, n$ is an integer. $\omega_{k, l}(y, t)$ is then defined as the function $\omega_{k}(y, t)$ that is solution of A1 with a stochastic forcing with only one Fourier component $(k, l)$. We then obtain

$$
\langle v \omega\rangle=\frac{1}{U^{\prime \prime}-\beta} \sum_{k, l} \frac{\hat{C}_{k, l}}{2}\left[2 \alpha\left\langle\left|\omega_{k, l}\right|^{2}\right\rangle-1\right],
$$

where the positive constants $\hat{C}_{k, l}$ are the Fourier coefficients of the spatial correlation function of the noise $\eta$, $\mathbb{E}\left[\eta(x, y, t) \eta\left(x^{\prime}, y^{\prime}, t^{\prime}\right)\right]=C\left(x-x^{\prime}, y-y^{\prime}\right) \delta\left(t-t^{\prime}\right)$. Be careful that in this formula the bracket $\left\langle\left|\omega_{k, l}\right|^{2}\right\rangle$ denotes a stochastic averaging, because the zonal average is already taken into account by the sum over all vector $k$.
The vorticity $\omega_{k, l}(y, t)$ is the solution of the stochastic partial differential equation

$$
\partial_{t} \omega_{k, l}+i k U \omega_{k, l}+i k\left(\beta-U^{\prime \prime}\right) \psi_{k, l}=-\alpha \omega_{k, l}+\eta_{l} .
$$

$\psi$ is the stream function defined through $\triangle \psi=\omega$. As the reader would have notice, we try to reduce the problem by expressing the solution as the sum of particular problems that we hope to be much simpler. Now we have to find an expression for $\omega_{k, l}$ instead of the full solution $\omega$. We will go one step further and show that the stochastic problem described by the two equations A2 A3 reduces in fact to a deterministic one, following 12. Equation (A3) can be formally written as

$$
\partial_{t} \omega_{k, l}+L_{k}\left[\omega_{k, l}\right]=-\alpha \omega_{k, l}+\eta_{l},
$$

where

$$
L_{k}\left[\omega_{k, l}\right]=i k U \omega_{k, l}+i k\left(\beta-U^{\prime \prime}\right) \psi_{k, l}
$$

is a linear operator for a given $U$. Then we use the fact that the noise $\eta_{k, l}$ is white in time and has an exponential correlation function $c_{l}(y)=e^{i l y}$ to express the quantity $\left\langle\left|\omega_{k, l}\right|^{2}\right\rangle$ as

$$
\left\langle\left|\omega_{k, l}\right|^{2}\right\rangle=\int_{-\infty}^{0} \mathrm{~d} t e^{2 \alpha t}\left|e^{t L_{k}}\left[c_{l}\right]\right|^{2}
$$

This formula should be understood as follows: $e^{-t L_{k}}\left[c_{l}\right]$ is the solution at time $t$ of the deterministic equation $\partial_{t} \omega_{d}+L_{k}\left[\omega_{d}\right]=0$ with initial condition $c_{l}:=y \rightarrow e^{i l y}$. The subscript $d$ will mean that we are dealing with the solution of a deterministic equation. The exponential $e^{2 \alpha t}$ ensures the convergence of this integral. The great advantage to have reduced the stochastic problem to a deterministic one is that we now have to solve an hydrodynamic problem, the propagation of a vorticity fluctuation in a shear flow, a problem for which much has already been done in the literature.

We thus have to solve the deterministic equation

$$
\partial_{t} \omega_{d}+i k U \omega_{d}+i k\left(\beta-U^{\prime \prime}\right) \psi_{d}=0,
$$

and we will take advantage of the small scale forcing limit where $K \rightarrow \infty$. We will consider the case of an infinite space in the $y$ direction, but we expect the result to be qualitatively similar for a finite space because it is sufficient that the scale of the forcing $\frac{1}{K}$ is small compared to the domain size, $\frac{1}{K} \ll L_{y}$. Please be careful here that $K$ and $k$ are different, $K^{2}=k^{2}+l^{2}$. We consider the limit $K$ infinite with both $k$ and $l$ going to infinity such that $l=k \tan \theta$ with $\theta$ a fixed constant. We will check that the limit $K \rightarrow \infty$ and $k \rightarrow \infty$ are not at all equivalent. As the leading order term in equation $\mathrm{A} 6$ is the free transport term $i k U \omega$, we will use the natural ansatz $\omega_{d}(y, t)=A_{k, l}(y, t) e^{-i k U(y) t}$ in equation A6. We obtain for $A_{k, l}$ the equation

$\partial_{t} A_{k, l}=-i \frac{U^{\prime \prime}-\beta}{k} \int \mathrm{d} Y H_{0}(Y) A_{k, l}\left(y-\frac{Y}{k}, t\right) e^{i k\left(U(y)-U\left(y-\frac{Y}{k}\right)\right) t}$, 
where $H_{0}$ is the Green function of the Laplacian operator $\left(\frac{\partial^{2}}{\partial y^{2}}-1\right)$ solving $\left(\frac{\partial^{2}}{\partial y^{2}}-1\right) H_{0}(y)=\delta(y)$. The Green function for the full Laplacian $\left(\frac{\partial^{2}}{\partial y^{2}}-k^{2}\right)$ writes $H_{k}(y)=\frac{1}{2|k|} H_{0}(|k y|)$. For infinite space, we have thus $H_{0}(y)=\frac{1}{2} e^{-|y|}$. This expression is very convenient to do asymptotic calculations because, as the reader can see, $\partial_{t} A_{k, l}$ is of order $\frac{1}{k}$ and thus goes to zero for large $k$. At zero order, we have only free transport of the perturbation, and the expression of $\omega$ is given by $\omega(y, t)=\omega(y, 0) e^{-i k U(y) t}$. The computation of the next order is more technical. From equation A5 we have the basic equality

$$
2 \alpha\left\langle\left|\omega_{k, l}\right|^{2}\right\rangle=2 \alpha \int_{0}^{+\infty} e^{-2 \alpha t}\left|A_{k, l}(y, t)\right|^{2} \mathrm{~d} t .
$$

Again, the bracket \langle\rangle is simply a stochastic averaging. An integration by parts gives

$2 \alpha\left\langle\left|\omega_{k, l}\right|^{2}\right\rangle=\left|A_{k, l}(y, 0)\right|^{2}+2 \mathcal{R} e \int_{0}^{+\infty} e^{-2 \alpha t} A_{k, l}(y, t)^{*} \partial_{t} A_{k, l}(y, t) \mathrm{d} t$.

Because $\left|A_{k, l}(y, 0)\right|^{2}=1$, it compensates exactly the -1 coming from the enstrophy injection in expression A2. Replacing $\partial_{t} A_{k, l}$ using A7 and equation A2 that gives the expression of $\mathcal{R} e\left\langle v_{k, l} \omega_{k, l} *\right\rangle$, we get the exact expression for the Reynolds stress divergence

$$
\mathcal{R} e\left\langle v_{k, l} \omega_{k, l}^{*}\right\rangle=\frac{\hat{C}_{k, l}}{k} \mathcal{I} m \int_{0}^{+\infty} \mathrm{d} t \ldots
$$$$
\ldots \int \mathrm{d} Y H_{0}(Y) A_{k, l}^{*}(y, t) A_{k, l}\left(y-\frac{Y}{k}, t\right) e^{i k\left(U(y)-U\left(y-\frac{Y}{k}\right)\right) t} e^{-2 \alpha t} \cdot \operatorname{R} e\left\langle v_{k, l} \omega_{k, l}^{*}\right\rangle \underset{K \uparrow \infty}{\sim} \frac{\hat{C}_{k, l}}{2 k} \mathcal{I} m \int \mathrm{d} Y \frac{e^{-|Y|} e^{-i Y \tan \theta}}{2 \alpha-i a Y+i b Y^{2}}
$$

Now we will have to use our hypothesis $K \rightarrow \infty$ to go on in the computation. Here comes a small subtlety, the $l$ component does not appears explicitly, it is hidden in the initial condition $A_{k, l}(y, 0)=e^{i l y}$. Therefore we cannot just expand $A_{k, l}\left(y-\frac{Y}{k}, t\right)$ in power of $\frac{1}{k}$ as one may guess at first sight. But expression (A7) tells us that $\partial_{t} A_{k, l} \rightarrow 0$ as $K$ goes to infinity. The right way to do the asymptotic expansion consists in an expansion of $A_{k, l}(y, t)$ wrt time and we use that each temporal derivation of $A_{k, l}$ is smaller of order $\frac{1}{K}$

$$
\begin{gathered}
A_{k, l}^{*}(y, t) A_{k, l}\left(y-\frac{Y}{k}, t\right)=A_{k, l}^{*}(y, 0) A_{k, l}\left(y-\frac{Y}{k}, 0\right) \ldots \\
\ldots+t \partial_{t}\left[A_{k, l}^{*}(y, t) A_{k, l}\left(y-\frac{Y}{k}, t\right)\right]_{t=0}+O\left(\frac{1}{K^{2}}\right)
\end{gathered}
$$

and we expand also $U$ using that $k$ is large

$$
\begin{aligned}
k\left(U(y)-U\left(y-\frac{Y}{k}\right)\right) & =U^{\prime}(y) Y-\frac{U^{\prime \prime}(y)}{2 k} Y^{2} \\
& :=a Y-b Y^{2} .
\end{aligned}
$$

The last expression defines $a$ and $b$. Using that $A_{k, l}(y, 0)=e^{i l y}, H_{0}(Y)=\frac{1}{2} e^{-|Y|}$ for infinite space, the first nonzero contribution is given by

$\mathcal{R} e\left\langle v_{k, l} \omega_{k, l}^{*}\right\rangle \underset{K \uparrow \infty}{\sim} \frac{\hat{C}_{k, l}}{2 k} \mathcal{I} m \iint \mathrm{d} Y \mathrm{~d} t e^{-|Y|} e^{-i Y \tan \theta} e^{i\left(a Y-b Y^{2}\right) t-2 \alpha t}$, with $\tan \theta=\frac{l}{k}$. One could check easily that the expression A8 is zero when $b=0$, that's why we have to keep the $b$ term to get the leading order term in the expansion in powers of $1 / K$.

We now prove that the second term in the expansion of $A_{k, l}^{*}(y, t) A_{k, l}\left(y-\frac{Y}{k}, t\right)$ is zero. With expression A7, we compute

$$
\partial_{t} A_{k, l}(y, 0)=-i \frac{U^{\prime \prime}-\beta}{k} \frac{e^{i l y}}{1+\tan ^{2} \theta},
$$

and then

$$
\begin{aligned}
& A_{k, l}^{*}(y, 0) \partial_{t} A_{k, l}\left(y-\frac{Y}{k}, 0\right)=-i \frac{U^{\prime \prime}-\beta}{k} \frac{e^{-i l \frac{Y}{k}}}{1+\tan ^{2} \theta} \\
& \partial_{t} A_{k, l}^{*}(y, 0) A_{k, l}\left(y-\frac{Y}{k}, 0\right)=i \frac{U^{\prime \prime}-\beta}{k} \frac{e^{-i l \frac{Y}{k}}}{1+\tan ^{2} \theta}
\end{aligned}
$$

The sum of both terms is zero. The next contribution then implies two derivatives and is of order $\frac{1}{K^{2}}$.

The presence of both exponentials in A8 allows us to invert the order of integration and integrate the time first. We get

$$
\mathcal{R} e\left\langle v_{k, l} \omega_{k, l}^{*}\right\rangle \underset{K \uparrow \infty}{\sim} \frac{\hat{C}_{k, l}}{2 k} \mathcal{I} m \int \mathrm{d} Y \frac{e^{-|Y|} e^{-i Y \tan \theta}}{2 \alpha-i\left(a Y-b Y^{2}\right)} .
$$

Now we have to use that $b:=\frac{U^{\prime \prime}}{2 k}$ is small and develop the denominator. This gives

$$
\underset{K \uparrow \infty}{\sim}-\frac{\hat{C}_{k, l}}{2 k} \mathcal{I} m \int \mathrm{d} Y b Y^{2} \frac{\partial}{\partial(a Y)}\left\{\frac{e^{-|Y|} e^{-i Y \tan \theta}}{2 \alpha-i a Y}\right\} .
$$

Now comes a trick: $U^{\prime \prime} Y \frac{\partial}{\partial\left(U^{\prime} Y\right)}=Y \frac{\partial\left(U^{\prime}(y) Y\right)}{\partial y} \frac{\partial}{\partial\left(U^{\prime}(y) Y\right)}=$ $Y \frac{\partial}{\partial y}$ and, recalling that $b=U^{\prime \prime} / 2 k$, we can put the derivative in $y$ in front of the integral. This is a method to recognize that our expression is a derivative in $y$ and get the analytic expression of the Reynolds stress. The idea to recognize a derivative seems to fall from nowhere, but it comes in fact from the work [15] and the expression they derived for the Reynolds stress. In Fourier space, the incompressibility condition writes $\mathcal{R} e\left\langle v_{k, l} \omega_{k, l}^{*}\right\rangle=$ $-\partial_{y} \mathcal{R} e\left\langle u_{k, l} v_{k, l}^{*}\right\rangle$, we then get

$$
-\partial_{y} \mathcal{R} e\left\langle u_{k, l} v_{k, l}^{*}\right\rangle \underset{K \uparrow \infty}{\sim}-\frac{\hat{C}_{k, l}}{4 k^{2}} \frac{\partial}{\partial y} \mathcal{I} m \int \mathrm{d} Y \frac{Y e^{-|Y|} e^{-i Y \tan \theta}}{2 \alpha-i a Y}
$$

and finally

$$
\mathcal{R} e\left\langle u_{k, l} v_{k, l}^{*}\right\rangle \underset{K \uparrow \infty}{\sim} C^{t e}+\frac{\hat{C}_{k, l}}{4 k^{2}} \mathcal{I} m \int \mathrm{d} Y \frac{Y e^{-|Y|} e^{-i Y \tan \theta}}{2 \alpha-i a Y} .
$$

The integration procedure defines $\langle u v\rangle$ up to a constant. This constant has no influence on the solution of the equation for the mean velocity profile, equation (4) in 


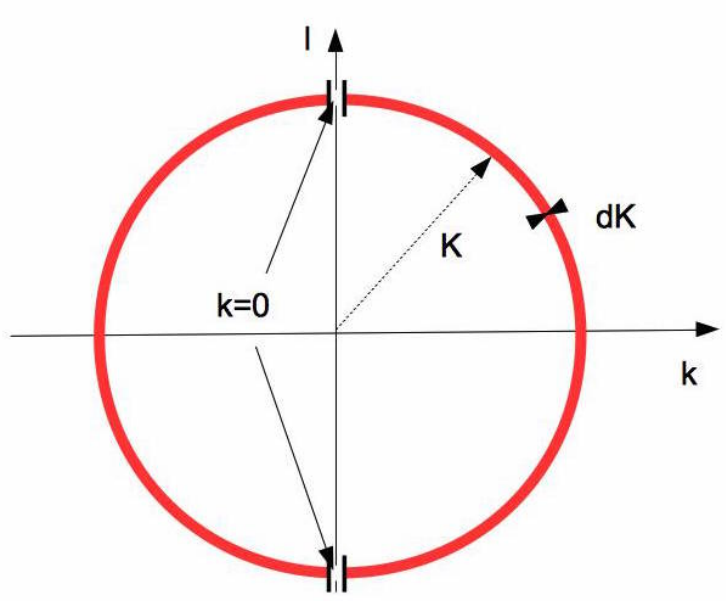

FIG. 5: Typical spectrum of the stochastic forcing

the article. In general, the expression of the Reynolds stress will depend on the boundary conditions.

Let us comment on this result. The parameter $\alpha$ is small because we have done the quasilinear approximation, but we keep it finite. It has a regularizing effect, and ensures that the integral remains well defined for every profile $U$ even if $U^{\prime}=0$ somewhere in the flow. The result above does not really makes sense for $\theta$ close to $\frac{\pi}{2}$ but the value $\frac{\pi}{2}$ is excluded because we assumed that the stochastic forcing does not inject energy in the mean flow. In numerical simulations, the spectrum of the stochastic forcing is often an annulus with a weight function to make it more or less anisotropic. The Fourier component $k=0$ is excluded because it corresponds to a direct stochastic forcing on the zonal flow (see figure (5)). To get results we can compare to simulations, we have to integrate the contributions to the Reynolds stress over the whole spectrum.

We will thus define a weight function $\hat{g}(\tan \theta) \operatorname{such}$ that the correlation function Fourier component is $\hat{C}_{k^{\prime}, l^{\prime}}=$ $\hat{g}(\tan \theta) K \delta\left(K^{\prime}-K\right)$. On figure (5) for example, we have a constant function $\hat{g}$ except around the excluded values $\frac{\pi}{2}$ and $-\frac{\pi}{2}$. We approximate the discrete sum in $\mathrm{A} 2$ by an integral assuming $\frac{1}{L_{x}} \ll \mathrm{d} K$. Because we have fixed the mean kinetic energy to 1 , the function $\hat{g}$ must satisfy

$$
\begin{aligned}
\frac{1}{2} \iint \mathrm{d} k^{\prime} \mathrm{d} l^{\prime} \frac{\hat{C}_{k^{\prime}, l^{\prime}}}{K^{\prime 2}} & =\frac{1}{2} \iint K^{\prime} \mathrm{d} K^{\prime} \mathrm{d} \theta \frac{\hat{g}(\tan \theta) K \delta\left(K^{\prime}-K\right)}{K^{\prime 2}} \\
& =\int_{-\infty}^{+\infty} \mathrm{d} x \frac{\hat{g}(x)}{1+x^{2}} \\
& =1,
\end{aligned}
$$

In the last integral, we have done the change of variable $\tan \theta=x$. Using this function to characterize the spectrum, we obtain the simple expression for the Reynold's stress

$$
\langle u v\rangle(y)=\pi \operatorname{Im} \int_{-\infty}^{+\infty} \frac{Y e^{-|Y|} g(-Y)}{\left(2 \alpha-i U^{\prime}(y) Y\right)} \mathrm{d} Y
$$

where $g$ is the inverse Fourier transform of $\hat{g}, g(Y)=$ $\frac{1}{2 \pi} \int \mathrm{d} x \hat{g}(x) e^{i Y x}$. Now that we have an expression for the Reynolds stress in terms of the mean profile $U$ we can solve the equation for the mean velocity profile and find the stationary solution, if any. We let $\chi$ be the function defined through $\chi(x):=\pi \operatorname{Im} \int_{-\infty}^{+\infty} \frac{Y e^{-|Y|} g(-Y)}{(1-i x Y)} \mathrm{d} Y$, and the stationary profile is defined by the set

$$
\begin{aligned}
\partial_{y}\langle u v\rangle & =-U \\
\langle u v\rangle & =\frac{1}{2 \alpha} \chi\left(\frac{U^{\prime}}{2 \alpha}\right) .
\end{aligned}
$$

The beautiful fact is that this system is integrable. If we replace $\langle u v\rangle$ in the first equation, it comes

$$
\frac{U^{\prime \prime}}{2 \alpha} \frac{1}{2 \alpha} \chi^{\prime}\left(\frac{U^{\prime}}{2 \alpha}\right)=-U
$$

so we can now multiply the equality by $U^{\prime}$ and integrate. Let $X$ be a primitive of $x \chi^{\prime}(x)$ and we have

$$
X\left(\frac{U^{\prime}}{2 \alpha}\right)+\frac{1}{2} U^{2}=C^{t e},
$$

this is equation 7 in the article.

\section{Appendix B: Expression of the Reynolds stress in the inertial limit}

The aim of this section is to explain how we can compute the Reynolds stress in the inertial limit $\alpha \rightarrow 0$ starting from the relation $\mathrm{A} 2$. We have to compute

$$
2 \alpha\left\langle\left|\omega_{k, l}\right|^{2}\right\rangle=2 \alpha \int_{-\infty}^{0} \mathrm{~d} t e^{2 \alpha t}\left|e^{t L_{k}}\left[c_{l}\right]\right|^{2},
$$

where $e^{-t L_{k}}\left[c_{l}\right]:=\omega_{d}$ is the solution to the deterministic equation

$$
\begin{aligned}
\partial_{t} \omega_{d}+i k U \omega_{d}+i k\left(\beta-U^{\prime \prime}\right) \psi_{d} & =0 \\
\left(\partial_{y}^{2}-k^{2}\right) \psi_{d} & =\omega_{d}
\end{aligned}
$$

with initial condition $c_{l}(y)=e^{i l y}$. We will first assume there are no neutral modes solutions of (B2). First, we do the change of time scale $2 \alpha t \rightarrow t$ in the integral of (B1). It gives us

$$
2 \alpha\left\langle|\omega|^{2}\right\rangle=\int_{-\infty}^{0} \mathrm{~d} t e^{t}\left|e^{\frac{t}{2 \alpha} L_{k}}\left[c_{l}\right]\right|^{2}
$$

When $\alpha$ goes to zero, the term $e^{\frac{t}{2 \alpha} L_{k}}\left[c_{l}\right]$ is the long time limit of the solution of $(\mathrm{B} 2)$. We use the nontrivial result for the case of non monotonous flows, of 22, that there exists a function $\omega_{d}^{\infty}(y)$ such that $\omega_{d}(y, t) \underset{t \rightarrow \infty}{\sim}$ $\omega_{d}^{\infty}(y) e^{-i k U t}$ when there are no neutral modes. Hence $\left|e^{\frac{t}{2 \alpha} L_{k}}\left[c_{l}\right]\right| \rightarrow\left|\omega_{d}^{\infty}(y)\right|$, and the presence of the exponential in the integral ensures the convergence of the whole. This proves that without neutral modes

$$
2 \alpha\left\langle|\omega|^{2}\right\rangle \underset{\alpha \rightarrow 0}{\longrightarrow}\left|\omega_{d}^{\infty}\right|^{2}
$$


How then can we compute the function $\omega_{d}^{\infty}$ ? We start from equation (B2) that describes the linear evolution of a perturbation $\omega(y, t) \mathrm{e}^{i k x}$ of meridional wave number $k$, and with streamfunction $\psi(y, t) \mathrm{e}^{i k x}$. The idea is to transform equation (B2) into an inhomogeneous Rayleigh equation, as classically done, and then to study its asymptotic solutions close to the real axis, which is the limit $\delta \rightarrow 0$, with the notations below. We introduce the function $\varphi_{\delta}(y, c)$ which is the Laplace transform of the stream function $\psi(y, t)$ i.e $\varphi_{\delta}(c):=$ $\int_{0}^{\infty} \mathrm{d} t \psi(y, t) e^{-i k(c+i \delta) t}$. The equation for $\varphi_{\delta}$ is

$$
\left(\frac{d^{2}}{d y^{2}}-k^{2}\right) \varphi_{\delta}(y, c)+\frac{\beta-U{ }^{\prime \prime}(y)}{U(y)-c-i \delta} \varphi_{\delta}(y, c)=\frac{\omega(y, 0)}{i k(U(y)-c-i \delta)},
$$

with the boundary conditions that $\varphi_{\delta}$ vanishes at infin- ity. We do not have a flow infinite in the $y$ direction, but as was already stated, the properties of the flow become local for large $K$. The choice to take vanishing boundary conditions at infinity is done for convenience and it is expected that this particular choice does not modify the physical behavior of the perturbation.

For all $\delta>0$ the function $\varphi_{\delta}$ is well defined. The inhomogeneous Rayleigh equation (B3) is singular for $\delta=$ 0 and for any critical point (or critical layer) $y_{c}$ such that the zonal flow velocity is equal to the phase speed: $U\left(y_{c}\right)=c$. One can show that $\varphi_{\delta}$ has a limit denoted $\varphi_{+}$when $\delta$ goes to zero. The function $\omega_{d}^{\infty}$ is then given by

$$
\omega_{d}^{\infty}(y)=i k\left(U^{\prime \prime}(y)-\beta\right) \varphi_{+}(y, U(y))+\omega(y, 0),
$$

see 22] for more details.
[1] L.D. Landau and E.M. Lifshitz. Fluid Mechanics. Number vol. 6. Elsevier Science, 2013.

[2] S.B. Pope. Turbulent flows, 2001.

[3] J. Paret and P. Tabeling. Experimental observation of the two-dimensional inverse energy cascade. Physical review letters, 79(21):4162, 1997.

[4] J. Sommeria. Experimental study of the two-dimensional inverse energy cascade in a square box. Journal of fluid mechanics, 170:139-168, 1986.

[5] DHE Dubin and TM ONeil. Two-dimensional guidingcenter transport of a pure electron plasma. Physical review letters, 60(13):1286, 1988.

[6] J.B. Marston, E. Conover, and T. Schneider. Statistics of an unstable barotropic jet from a cumulant expansion. Journal of Atmospheric Sciences, 65:1955, 2008.

[7] P.H. Diamond, S.I. Itoh, K. Itoh, and T.S. Hahm. Zonal flows in plasma review. Plasma Physics and Controlled Fusion, 47(5):R35, 2005.

[8] B. Galperin, R. Young, S. Sukoriansky, N. Dikovskaya, P. Read, A. Lancaster, and D. Armstrong. Cassini observations reveal a regime of zonostrophic macroturbulence on jupiter. Icarus, 229:295-320, 2014.

[9] F. Bouchet and E. Simonnet, Random changes of flow topology in two-dimensional and geophysical turbulence. Physical review letters, 102(9), 094504, 2009.

[10] A. Frishman, J. Laurie and G. Falkovich. Jets or vortices? What flows are generated by an inverse turbulent cascade? Physical Review Fluids, volume 2, 2017.

[11] B. Farrell and P.Ioannou. Structural stability of turbulent jets. Journal of the atmospheric sciences, 60(17):2101-2118, 2003.

[12] F. Bouchet, C. Nardini, and T. Tangarife. Kinetic theory of jet dynamics in the stochastic barotropic and $2 \mathrm{~d}$ navier-stokes equations. Journal of Statistical Physics, 153(4):572-625, 2013.

[13] J. Laurie, G. Boffetta, G. Falkovich, I. Kolokolov, and V. Lebedev. Universal profile of the vortex condensate in two-dimensional turbulence. Physical review letters, 113(25):254503, 2014.

[14] G. Falkovich. Interaction between mean flow and turbulence in two dimensions. In Proc. R. Soc. A, volume 472, page 20160287. The Royal Society, 2016.

[15] K. Srinivasan and WR Young. Reynolds stress and eddy diffusivity of $\beta$-plane shear flows. Journal of the Atmospheric Sciences, 71(6):2169-2185, 2014.

[16] I. Kolokolov and V. Lebedev. Velocity statistics inside coherent vortices generated by the inverse cascade of $2 d$ turbulence. arXiv preprint arXiv:1609.00918, 2016

[17] I.Kolokolov and V. Lebedev. Structure of coherent vortices generated by the inverse cascade of two-dimensional turbulence in a finite box. Physical Review E, volume 93, 033104, 2016.

[18] T.Tangarife. Kinetic theory and large deviations for the dynamics of geophysical flows. https://tel.archivesouvertes.fr/tel-01241523, These Ecole normale supérieure de lyon, 2015.

[19] C.Nardini and T. Tangarife. Fluctuations of largescale jets in the stochastic 2D Euler equation. arXiv:1602.06720, 2016.

[20] C.Porco, R.West, A.McEwen, A. Del Genio, A. Ingersoll, P. Thomas, S. Squyres, L. Dones, C. Murray, T. Johnson, et al. Cassini imaging of jupiter's atmosphere, satellites, and rings. Science, 299(5612):1541-1547, 2003.

[21] Colette Salyk, Andrew P Ingersoll, Jean Lorre, Ashwin Vasavada, and Anthony D Del Genio. Interaction between eddies and mean flow in jupiter's atmosphere: Analysis of cassini imaging data. Icarus, 185(2):430-442, 2006.

[22] F. Bouchet and H. Morita. Large time behavior and asymptotic stability of the 2D Euler and linearized Euler equations. Physica D Nonlinear Phenomena, 239:948966, June 2010.

[23] N. Constantinou. Formation of large-scale structures by turbulence in rotating planets. arXiv preprint arXiv:1503.07644, 2015. 